\title{
SUPPLEMENT
}

\author{
Sümeyra Altan ${ }^{1}$ \\ Bercim Berberoglu ${ }^{2}$ \\ Sinan Canan ${ }^{3}$ \\ Şenol Dane ${ }^{4}$
}

Turgut Özal University, Medical School ${ }^{1}$, nBeyin-Ankara ${ }^{2}$, Medipol University, Medical Faculty, Department of Physiology ${ }^{3}$, Turgut Özal Universitity, Medical School, Department of Physiology ${ }^{4}$

\section{Effects of neurofeedback therapy in healthy young subjects}

\begin{abstract}
Purpose: Neurofeedback refers to a form of operant conditioning of electrical brain activity, in which desirable brain activity is rewarded and undesirable brain activity is inhibited. The research team aimed to examine the efficacy of neurofeedback therapy on electroencephalogram (EEG) for heart rate, electrocardiogram (ECG) and galvanic skin resistance (GSR) parameters in a healthy young male population.

Methods: Forty healthy young male subjects aged between 18 to 30 years participated in this study. Neurofeedback application of one session was made with bipolar electrodes placed on $\mathrm{T}_{3}$ and $\mathrm{T}_{4}$ (temporal 3 and 4 ) regions and with reference electrode placed on $\mathrm{PF}_{1}$ (prefrontal 1). Electroencephalogram (EEG), electrocardiogram (ECG) and galvanic skin resistance (GSR) were assessed during Othmer neurofeedback application of one session to regulate slow wave activity for forty minutes thorough the session. Data assessed before neurofeedback application for 5 minutes and during neurofeedback application of 30 minutes and after neurofeedback application for 5 minutes throughout the session of 40 minutes. Means for each 5 minutes, that is to say, a total 8 data points for each subjects over 40 minutes, were assessed.
\end{abstract}

Results: Galvanic skin resistance increased and heart rate decreased after neurofeedback therapy. Beta activity in EEG increased and alfa activity decreased after neurofeedback therapy.

Conclusions: These results suggest that neurofeedback can be used to restore sympathovagal imbalances. Also, it may be accepted as a preventive therapy for psychological and neurological problems.

\section{Correspondence to:}

Senol Dane

Turgut Ozal University, Faculty of Medicine, Department of Medical Physiology Yunus Emre Cad., Takdir Sok No:5, Yenimahalle, Ankara, 06170, TURKEY

E-mail: sdane@turgutozal.edu.tr 
Neurofeedback refers to a form of operant conditioning of electrical brain activity, in which desirable brain activity is rewarded and undesirable brain activity is inhibited [1]. Neurofeedback is believed to elicit growth and changes at the cellular levels of the brain, which, in turn, support brain functioning and behavioral cognitive performance [2].

Neurofeedback training is useful in treatment of different disorders in both adults and children. Positive effects of neurofeedback in adults, for example, have been found for Attention Deficit Hyperactivity Disorder [3], traumatic brain injury [4], epilepsy [5], depression [6], migraine [7], addiction [8], anxiety disorders [9] and general cognitive performance [10]. Neurofeedback protocols that aim at inhibiting theta activity while rewarding beta activity have led to successful

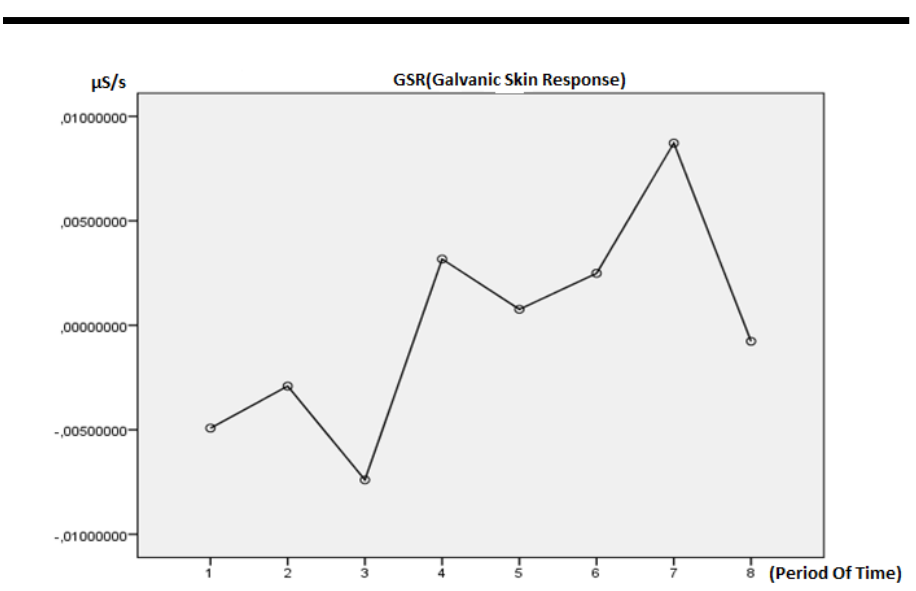

FIGURE 1. Changes in the galvanic skin resistance over time (eight 5 minute measurements over a 40 minute session).

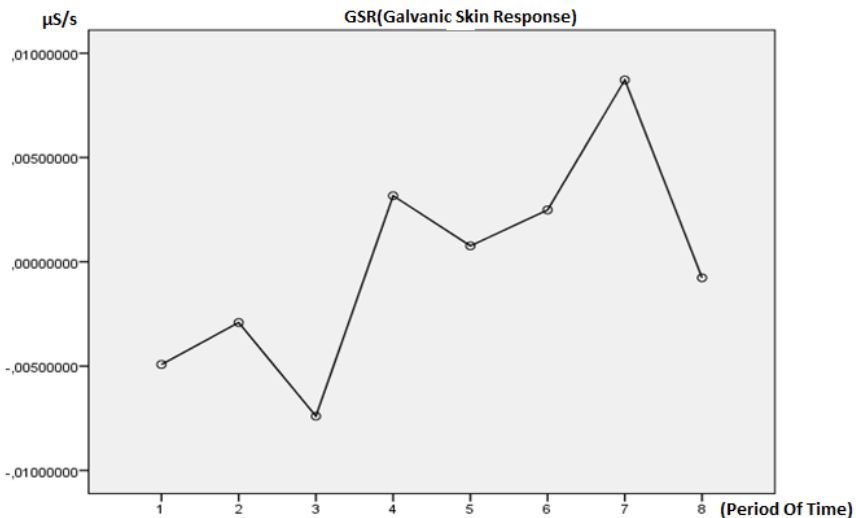

FIGURE 2. Changes in the heart rate over time (eight 5 minute measurements over a 40 minute session). alleviation of symptoms associated with ADHD, such as deficits in sustained attention, impulsivity and control over hyperactive behaviors (reviews in Butnik, 2005; Fox, Tharp, \& Fox, 2005).

In the present study, the effects of neurofeedback therapy on electroencephalogram (EEG), electrocardiogram (ECG) and galvanic skin resistance (GSR) parameters were investigated in a healthy young male population.

\section{Materials and Methods}

\section{Subjects}

Forty healthy young male subjects aged between 18 to 30 years participated in this study. Exclusion criteria were health

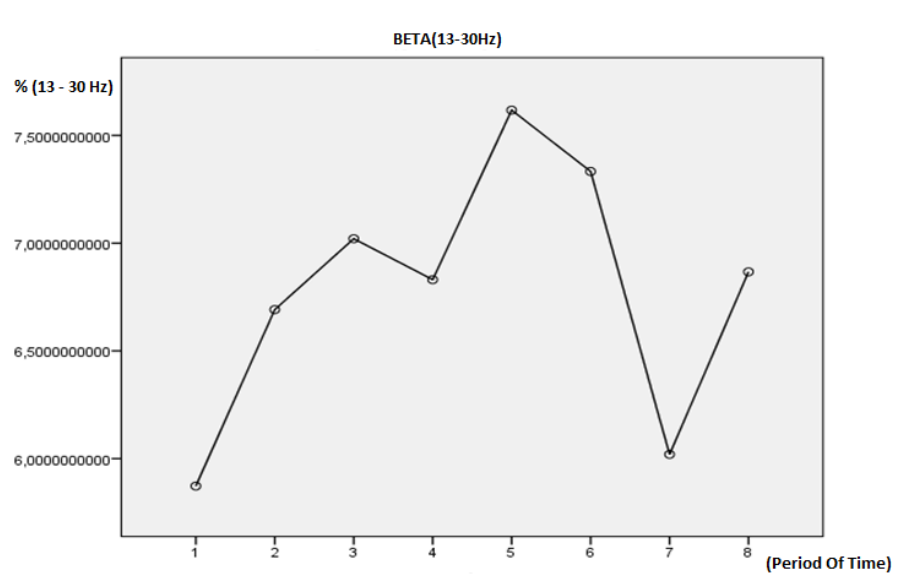

FIGURE 3. Changes in the beta activity over time (eight 5 minute measurements over a 40 minute session).

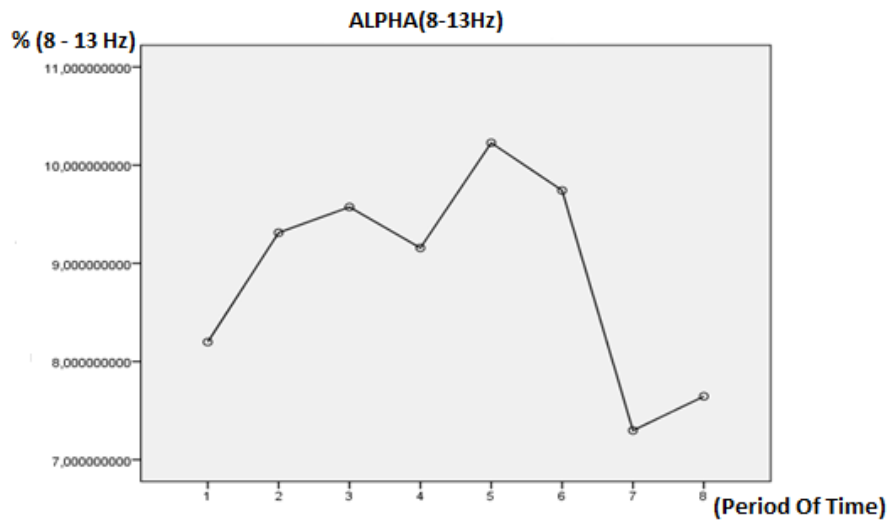

FIGURE 4. Changes in the alfa activity over time (eight 5 minute measurements over a 40 minute session). 
problems such as psychiatric, respiratory, metabolic, cardiac or autonomic nervous system diseases. The Ethical Committee of the Faculty of Medicine of the University of Turgut Ozal approved this study.

\section{Neurofeedback Therapy}

Neurofeedback application of one session was made with bipolar electrodes placed on $\mathrm{T}_{3}$ and $\mathrm{T}_{4}$ (temporal 3 and 4) regions and with reference electrode placed on $\mathrm{PF}_{1}$ (prefrontal 1). Electroencephalogram (EEG), electrocardiogram (ECG) and galvanic skin resistance (GSR) were assessed during Othmer neurofeedback application of one session to regulate slow wave activity for forty minutes thorough the session. All subjects were shown the first 40 minutes of the movie "The Shawshank Redemption" throughout the neurofeedback session. Neurofeedback application duration was 30 minutes for all subjects. EEG. ECG and GSR were assessed 1) for 5 minutes before neurofeedback application, 2) during neurofeedback application of 30 minutes and 3) after neurofeedback application for 5 minutes throughout the 40 minute session. Means for each 5 minutes, that is to say, a total of 8 data points for each subjects over the 40 minute session, were assessed.

For statistical analysis, IBM SPSS Statistics v21 was used. Descriptive statistics are done for demographic data analysis. Kolmogorov-Smirnov test was used to determine the normalization of data distribution. Mauchly's Test of Sphericity was used to calculate the difference among averages of eight different periods. For statistical significance p-value $<$ 0.05 was used.

\section{Results}

Galvanic skin resistance increased significantly after neurofeedback therapy compared with before therapy $(p<0.05)$ (see Figure 1). Heart rate decreased significantly after neurofeedback therapy compared with before therapy $(\mathrm{p}<0.05)$ (see Figure 2). Beta activity in EEG increased significantly after neurofeedback therapy compared to before therapy $(\mathrm{p}<0.05)$ (see Figure 3). Alfa activity in EEG decreased significantly after neurofeedback therapy compared with before therapy $(\mathrm{p}<0.05)$ (see Figure 4$)$. Delta, theta and gamma activities in EEG were not statistically significant before and after neurofeedback therapy.

\section{Discussion}

In the present study, statistically significant effects of neurofeedback therapy on EEG, ECG and GSR parameters in a healthy young male population were found. Increased galvanic skin resistance and decreased heart rate may be accepted as the possible effects of selective sympathetic stimulation via the neurofeedback therapy.

Neurofeedback therapy can be used for a wide range of neurologic and psychiatric problems [3-10]. The results of this study suggest that neurofeedback can be used to restore the sympathovagal imbalances, for which wet cupping and reflexological therapies have also been used $[11,12]$. It may be accepted as a preventive therapy application for psychological and neurological problems. These results also suggest that neurofeedback therapy increases beta activity in EEG; causing increased attention and noesis. Because neurofeedback is a noninvasive methodology, it may be used to facilitate learning and instruction.

\section{References}

1. Kouijzer MEJ, de Moor JMH, Gerrits BJL, Congedo M, van Schie HT. Neurofeedback improves executive functioning in children with autism spectrum disorders. Research in Autism Spectrum Disorders 2009; 3: 145-162.

2. Demos JN. Getting Started with Neurofeedback. New York: WW Norton \& Company Inc 2005.

3. Kropotov JD, Grin-Yatsenko VA, Ponomarev VA, Chutko LS, Yakovenko EA, Nikishena IS. ERP correlates of EEG relative beta training in ADHD Children. International Journal of Psychophysiology 2005; 55: 23-34.

4. Thornton K. Improvement/rehabilitation of memory functioning with neurotherapy/QEEG biofeedback. Journal of Head Trauma Rehabilitation 2000; 15: 1285-1296.

5. Sterman MB. Basic concepts and clinical findings in the treatment of seizure disorders with EEG operant conditioning. Clinical Electroencephalography 2000; 31: 45-55.

6. Hammond DC. QEEG-guided neurofeedback in the treatment of obsessive-compulsive disorder. Journal of Neurotherapy 2003; 7: 25-52.

7. Kropp P, Siniatchkin M, Gerber WD. On the pathophysiology of migraine-Links for "empirically based treatment" with neurofeedback. Applied Psychophysiology and Biofeedback 2002; 27(3): 203-213.

8. Trudeau DL. Applicability of brain wave biofeedback to substance use disorder in adolescence. Child and Adolescent Psychiatric Clinics of North America 2005; 14: 125-136.

9. Moore NC. A review of EEG biofeedback treatment of anxiety disorders. Clinical Electroencephalography 2000; 31: 1-6. 
10. Vernon D, Egner T, Cooper N, Compton T, Neilands C, Sheri A, et al. The effect of training distinct neurofeedback protocols on aspects of cognitive performance. International Journal of Psychophysiology 2003; 47: 75-85.

11. Arslan M, Yesilcam N, Aydin D, et al. Wet cupping therapy restores sympathovagal imbalances in cardiac rhythm. Journal of Alternative and Complementary Medicine 2014; 20 (4): 318-321.

12. Isik B, Aydin D, Arslan M, et al. Reflexological therapy induces a state of balance in autonomic nervous system. Clinical and Investigative Medicine 2015; 38(4): E244-E248. 